\title{
Devons-nous avoir peur des DRG?
}

L'évolution incessante des coûts hospitaliers n'a longtemps que peu préoccupé le corps médical. Cependant, depuis une dizaine d'années, les autorités politiques ont pris diverses mesures pour enrayer cette augmentation. Conjointement avec la montée en puissance des assureurs, il en est résulté une pression accrue sur les médecins-cadres hospitaliers.

L'introduction de SwissDRG, une structure tarifaire unifiée basée sur les diagnostics et les procédures, est voulue par le législateur. De nombreux pays pratiquent une telle manière de faire depuis longtemps et leur expérience nous est précieuse. L'article de R. O. Binswanger publié dans ce numéro du BMS présente un inventaire des phénomènes récemment observés en Allemagne suite à la mise en œuvre des G-DRG (les DRG allemands) et de la délégation budgétaire aux directeurs de clinique. Le parallélisme avec la Suisse est intéressant et légitime. Néanmoins, il faut considérer que les effets de SwissDRG seront plus progressifs dans notre pays, principalement en raison de notre structure décentralisée avec 26 systèmes de santé autonomes, la majorité des cantons ayant déjà introduit un financement hospitalier par DRG avec toutes ses conséquences. Il n'y aura donc pas de changement abrupt pour tout un pays, le jour où SwissDRG entrera en vigueur.

D'autre part, il est essentiel que le corps médical prenne conscience du rôle qu'il a à jouer dans la gestion finan- cière de nos établissements hospitaliers. Les médecinscadres doivent s'y intéresser mais doivent aussi recevoir de leur direction le support administratif indispensable pour pouvoir relever ce défi. Au niveau de la FMH, nous avons d'emblée estimé que l'élaboration, puis le contrôle et l'entretien de la structure tarifaire SwissDRG ne peuvent se faire qu'avec les médecins. Partenaires du projet à part entière, nous y développons une politique offensive, ayant l'ambition de garder notre liberté thérapeutique et le contrôle de la qualité de nos prestations sans être les otages des impératifs financiers.

Le monde hospitalier helvétique change de plus en plus rapidement. Qu'ils le veuillent ou non, les médecins travaillant à l'hôpital sont pris dans un contexte global où tout s'imbrique, se contrôle et se développe, dans une alchimie instable de médecine, de fibre sociale, d'économie et d'informatique. Baisser les bras face à ces réalités est indigne de notre corporation. Nous devons garder la conduite des affaires, faire sans cesse valoir notre point de vue, participer à la prise des décisions difficiles, afin de conserver la stabilité du système et de rester crédibles face à nos patients aussi bien que vis-à-vis de nos collaborateurs.

Dr Pierre-François Cuénoud, membre du Comité central de la FMH 